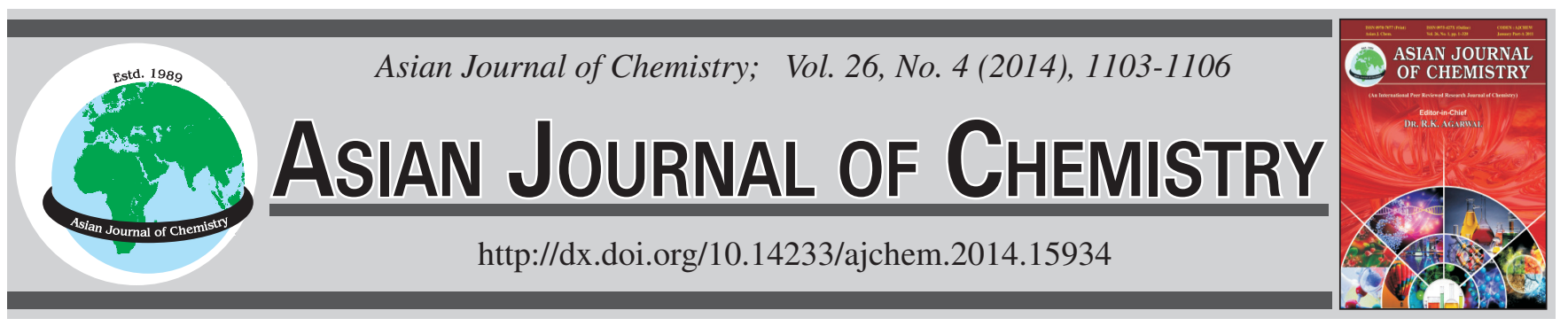

\title{
Flocculation Behavior in Removal of Dimethyl Phthalate from Water using Cationic Flocculant P(AM-DAC-DMC)
}

\author{
JUNREN ZHU ${ }^{1}$ and ZHENZHEN JIANG ${ }^{2, *}$
}

${ }^{1}$ Chongqing City Management College, Chongqing 401331, P.R. China

${ }^{2}$ Chongqing Vocational Institute of Engineering, Chongqing 400037, P.R. China

*Corresponding author: Tel: +86 23 63311221; E-mail: livelyzeng@ 126.com

Flocculation process with $\mathrm{P}(\mathrm{AM}-\mathrm{DAC}-\mathrm{DMC})$ as a novel flocculant was used for the removal of dimethyl phthalate from water. The structures and morphologies of $\mathrm{P}(\mathrm{AM}-\mathrm{DAC}-\mathrm{DMC})$ was characterized by nuclear magnetic resonance spectra $\left({ }^{1} \mathrm{H}\right.$ NMR) and scanning electron microscope. In order to maximize the removal efficiency of dimethyl phthalate, single factor experiment was investigated with the factors of dosage, initial $\mathrm{pH}$ value, settling time and molecular weight. Furthermore, an orthogonal array L9 $\left(3^{3}\right)$ were employed to optimize the factors of dosage, initial $\mathrm{pH}$ value and settling time. The results showed that the optimal conditions were dosage of $9.0 \mathrm{mg} \mathrm{L}^{-1}$, initial $\mathrm{pH}$ value of 6.0 and settling time of $3 \mathrm{~h}$. In addition, the removal rate of dimethyl phthalate reached a maximum value being $94.6 \%$.

Keywords: Cationic polyacrylamide, Dimethyl phthalate, Water treatment, Flocculation process, Orthogonal array design.

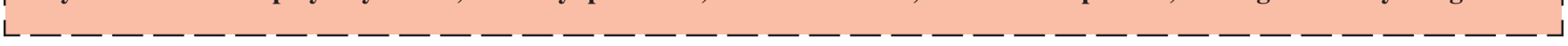

\section{INTRODUCTION}

Phthalate esters (PAEs) has been widely used as an important chemical raw material in plastic industrial applications to improve the mechanical properties of the plastic resin, particularly its flexibility ${ }^{1,2}$. Dimethyl phthalate ester (DMP), a short-chained ester among phthalate esters, is one of the most important varieties ${ }^{3,4}$. Dimethyl phthalate ester is essentially non-covalently bound to resin and is therefore able to migrate into the environment. Because of its common usage and its refractory biodegradability, it has been detected in many natural environments such as fresh waters, marine waters, landfill leachate, sewage sludge and sediments ${ }^{5,6}$. Furthermore, DMP can be bioconcentrated and biomagnified in the food chain through water ${ }^{7}$. However, the long-term exposure to DMP can cause various etiological diseases in the nervous system and gland cancers of animals. It is also suspected to dysfunction of endocrine system and result in decreased fertility in human beings ${ }^{8}$. Therefore, because of its ecological toxicity and hazard, DMP has been designated as a priority organic pollutant by the United States Environmental Protection Agency (US EPA) ${ }^{9}$.

Literature reported a number of methods for removing phthalate esters from an aqueous environment. the study have showed that the resin absorption method is better effect than others and has much more adsorbent $\operatorname{costs}^{10}$. Moreover, dimethyl phthalate ester are difficult to degrade biologically and photo-chemically. Dimethyl phthalate ester, negatively charged particles, is considered to easily remove from water through Coagulation-flocculation process ${ }^{11}$. Coagulationflocculation process is an important water pollution control technology that finds a wide range of application in water or wastewater treatment facilities ${ }^{12,13}$. The flocculating effect is determined mainly by the qualities and species of flocculants ${ }^{14}$. P(AM-DMC-DAC), one kind of ternary copolymerized cationic polyacrylamide, was synthesized using acrylamide (AM), methacryloxyethyltrimethyl ammonium chloride (DMC) and acryloxyethyltrimethyl ammonium chloride (DAC) by photopolymerization technique. P(AM-DMC-DAC) as one novel flocculant has become used in wastewater and sludge dewater treatment ${ }^{15,16}$. Therefore, flocculation process is worth investigating the possibility of the same being successful to deal with dimethyl phthalate ester in water using P(AM-DMC-DAC).

In the study, the structure and morphologies of P(AMDMC-DAC) was characterized by nuclear magnetic resonance spectra ( ${ }^{1} \mathrm{H}$ NMR) and scanning electron microscopy (SEM). Secondly, To maximize the removal efficiency of DMP, single factor technique was investigated with the factors of dosage, initial $\mathrm{pH}$ value, settling time and molecular weight. Lastly, an orthogonal array L9 $\left(3^{3}\right)$ optimizing the factors of dosage, initial $\mathrm{pH}$ value and settling time were also studied.

\section{EXPERIMENTAL}

Analytical grade dimethyl phthalate was purchased from Chongqing Oriental reagent factory; HPLC grade methanol 
was purchased from Shandong yuwang industrial Co. Ltd.; de-ionized water was madeself in the lab and $\mathrm{pH}$ value of solution was adjusted using $\mathrm{NaOH}$ and $\mathrm{HCl}$ solution.

Instruments used in the experimental setup were as follows: AVANCE 500 nuclear magnetic resonance spectrometer (NMR) from BRUKER Company (Germany); VEGA II LMU scanning electron microscope (SEM) from TES-CAN Company (Czech); ZR4-6 Jar Tester supplied by Zhongrun Water Industry Technology Development Co. Ltd. (Shenzhen, China); high performance liquid chromatograph (Waters e2695) supplied by Water Company (USA).

Characteristics: The copolymer was purified using acetone for three times in order to obtain the pure solid of $\mathrm{P}(\mathrm{AM}-\mathrm{DMC}-\mathrm{DAC})$. Then the copolymer was dried at $60{ }^{\circ} \mathrm{C}$ in an oven for several days. SEM images were taken through scan electronic microscope. The powdered product was dissolved with deuterium oxide $\left(\mathrm{D}_{2} \mathrm{O}\right)$ as the solvent, before analyzes using ${ }^{1} \mathrm{H}$ NMR.

Wastewater sample: $1 \mathrm{~g}$ of DMP was placed in a 1000 $\mathrm{mL}$ volumetric flask, dissolved in methanol and then constant volume to the mark with distilled water and prepared 1000 $\mathrm{mg} \mathrm{L}^{-1}$ a single standard stock solution, stored in a refrigerator at $4{ }^{\circ} \mathrm{C}$ and set aside. Take $0.5 \mathrm{~mL}$ DMP single standard stock solution was placed in a $1000 \mathrm{~mL}$ volumetric flask, then constant volume to the mark with distilled water and diluted $0.5 \mathrm{mg} \mathrm{L}^{-1}$ simulation water solution.

Flocculation experiments: The coagulation-flocculation experiments were carried out using a program-controlled jar test apparatus (ZR4-6) at room temperature. $500 \mathrm{~mL}$ of 0.5 $\mathrm{mg} \mathrm{L}^{-1}$ DMP simulation water solution was transferred into a beaker. Flocculants were dosed under rapid stirring speed of $300 \mathrm{rpm}$ for $1 \mathrm{~min}$ and changed to the medium stirring speed of $160 \mathrm{rpm}$ for $4 \mathrm{~min}$, then the speed was reduced to a slow stirring speed of $40 \mathrm{rpm}$ for $5 \mathrm{~min}$. After quiescent settling for $2 \mathrm{~h}$, the cleared water solution was extracted to measure the residual DMP using HPLC and the removal efficiency (RE) of DMP was calculated using the following eqn. 1 , where $C_{1}$ and $\mathrm{C}_{2}$ are the initial and final concentration of DMP, respectively.

$$
\operatorname{RE}(\%)=\frac{\mathrm{C}_{1}-\mathrm{C}_{2}}{\mathrm{C}_{1}} \times 100 \%
$$

Orthogonal array design (OAD): An orthogonal array L9 $\left(3^{3}\right)$ (eight degrees of freedom) was used to analyze the significance of each factor. Table- 1 showed the three factors at three levels for the OAD, with a dummy factor, D.

\begin{tabular}{cccc}
\multicolumn{4}{c}{ TABLE-1 } \\
FACTORS AND LEVELS ESTABLISHMENT \\
\hline Level & Dosage $\left(\mathrm{mg} \mathrm{L}^{-1}\right)$ & $\mathrm{pH}$ & Settling time (h) \\
\hline 1 & 8 & 5 & 2 \\
2 & 9 & 6 & 3 \\
3 & 10 & 7 & 4 \\
\hline
\end{tabular}

\section{RESULTS AND DISCUSSION}

Characterization of copolymer: The possible chemical composition of the copolymer was investigated using a ${ }^{1} \mathrm{H}$ NMR. Fig. 1 showed ${ }^{1} \mathrm{H}-\mathrm{NMR}$ spectrum of $\mathrm{P}(\mathrm{AM}-\mathrm{DAC}-\mathrm{DMC})$ being molecular weight $1.2 \times 10^{7}$. It can be seen from Fig. 1 that the resonance peak at $\delta=4.80 \mathrm{ppm}$ which was attributed to the solvent $\mathrm{D}_{2} \mathrm{O}$. The peaks at $\delta=2.23$ and $\delta=1.63$ ppm were derived from the proton at the methane group and methylene group of $-\mathrm{CH}_{2}-\mathrm{CH}-\mathrm{CONH}_{2}$ in the acrylonitrile. Furthermore, the resonance peak at $\delta=3.18$ and $\delta=3.78$ was ascribed to the proton at the methyl group and methylene group of $-\mathrm{N}^{+}$in the DAC and DMC. The result is broadly in line with the composition of self-made $\mathrm{P}(\mathrm{AM}-\mathrm{DAC}-\mathrm{DMC})$.

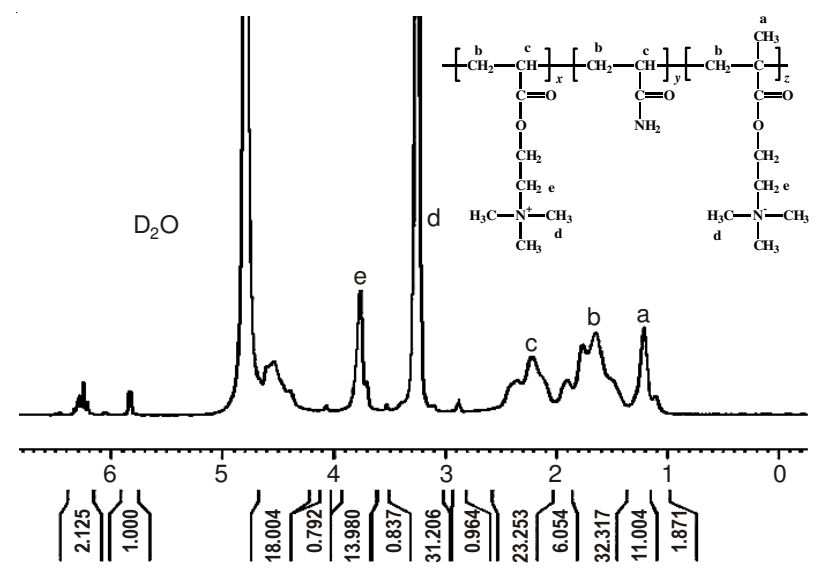

Fig. 1. ${ }^{1} \mathrm{H}-\mathrm{NMR}$ spectra of AM-DMC-DAC copolymer

Fig. 2 showed network structure of floccules with lots of small holes and a few cavities were observed in the P(AMDAC-DMC). The conclusions were drawn based on previous studies that the gel network structure was more favorable to coagulate colloidal particles compared with the smooth structure $^{17}$. Therefore, P(AM-DMC-DAC) had the advantages of adsorption-bridging and enmeshment in removing DMP from water.

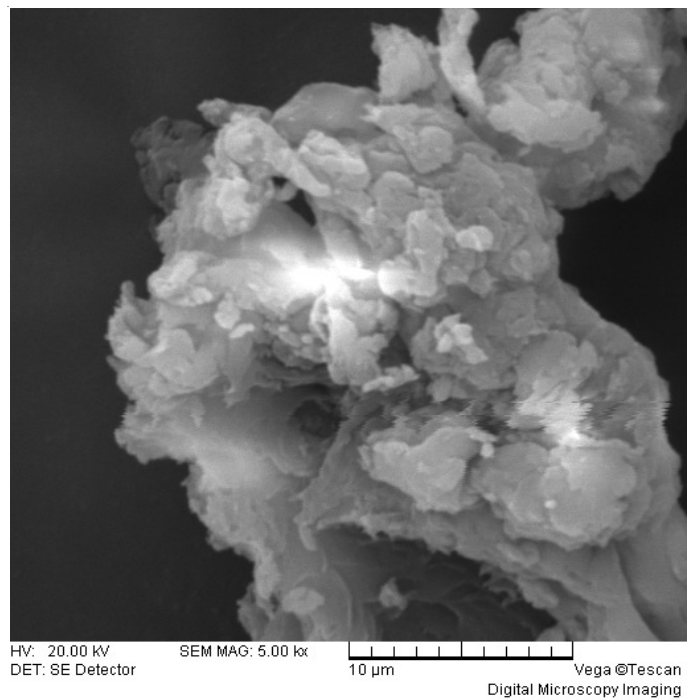

Fig. 2. SEM micrographs of P(AM-DAC-DMC)

Effect of dosage on removal rate of DMP: The effect of $\mathrm{P}(\mathrm{AM}-\mathrm{DAC}-\mathrm{DMC})$ dosage on the removal efficiency of DMP was investigated with the dosage range from 7 to $12 \mathrm{mg} \mathrm{L}^{-1}$ and not adjust the $\mathrm{pH}$ value. Fig. 3 showed that the maximum DMP removal rate at $91.4 \%$ can be observed while dosage is controlled at $9 \mathrm{mg} \mathrm{L}^{-1}$. The results indicated that flocculants dosage less than $9 \mathrm{mg} \mathrm{L}^{-1}$ is not enough charge neutralization 
between flocculants and DMP particles. However, DMP particles can be complete reversal by the flocculants charge under excessive dosage, leading to DMP particles fail to adsorptionbridging. Therefore, the adopted optimum dosage for removal of DMP is $9 \mathrm{mg} \mathrm{L}^{-1}$.

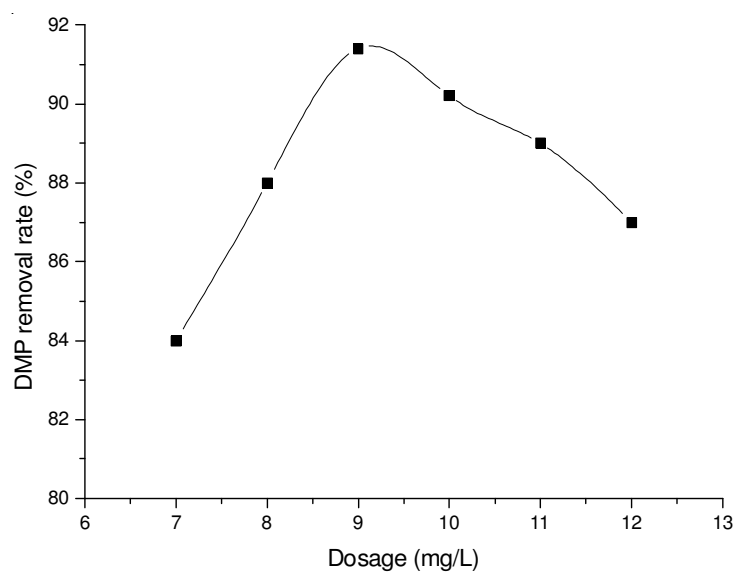

Fig. 3. Effect of dosage on removal of DMP

Effect of pH on removal rate of DMP: The effect of $\mathrm{pH}$ on the removal efficiency of DMP was investigated with the $\mathrm{pH}$ value from 2 to 10 and the dosage at $9 \mathrm{mg} \mathrm{L}^{-1}$. Fig. 4 showed the maximum DMP removal rate at $92.2 \%$ can be observed while initial $\mathrm{pH}$ was 6 . The reason was mainly due to the wastewater $\mathrm{pH}$ value impacting on the Zeta potential of the surface charge of colloidal particle and the hydrolysis product of flocculants. P(AM-DMC-DAC) used in this experiment carried a positive charge and when dissociating in water, the complete charge neutralization are needed to less flocculants under weak acid conditions. However, $\mathrm{pH}$ less than 6 can make DMP particles easy charge reversal, leading to DMP particles fail to adsorption-bridging. Therefore, the adopted optimum $\mathrm{pH}$ for DMP removal is 6 .

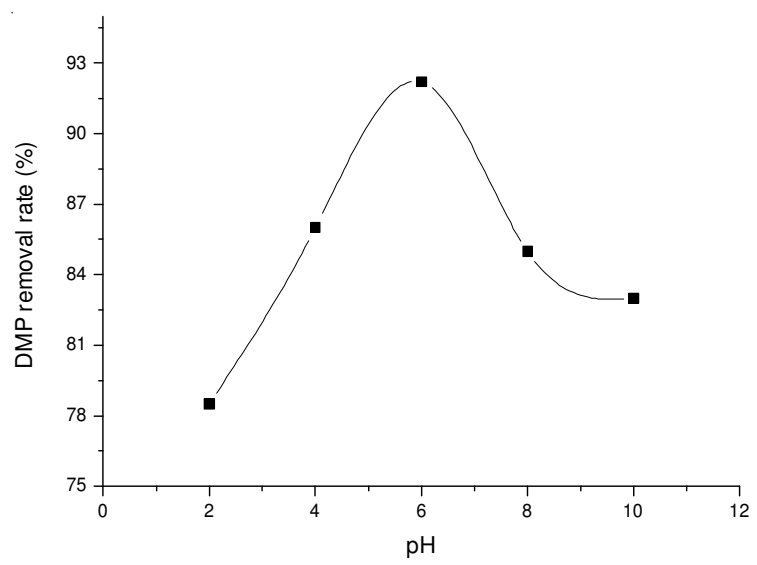

Fig. 4. Effect of $\mathrm{pH}$ on removal of DMP

Effect of settling time on removal rate of DMP: The effect of settling time on the removal efficiency of DMP was investigated with the settling time from $0.5-4 \mathrm{~h}$, the dosage at $9 \mathrm{mg} \mathrm{L}^{-1}$ and not adjust the $\mathrm{pH}$ value. Fig. 5 showed the maximum DMP removal rate at $93.8 \%$ can be observed while settling time was $3 \mathrm{~h}$. It can be seen that DMP removal efficiency increased from 78.3 to $93.8 \%$ while the settling time increased from $0.5 \mathrm{~h}$ to $3 \mathrm{~h}$. However, the DMP removal efficiency reduction more than settling $3 \mathrm{~h}$. The possible reason is that the longer settling time increase enmeshment and sedimentation opportunities between flocs and pollutants particles. However, DMP molecule can be released from flocs through the thermal motion of the molecules after stabilize floc. Therefore, the adopted optimum settling time for DMP removal is $3 \mathrm{~h}$.

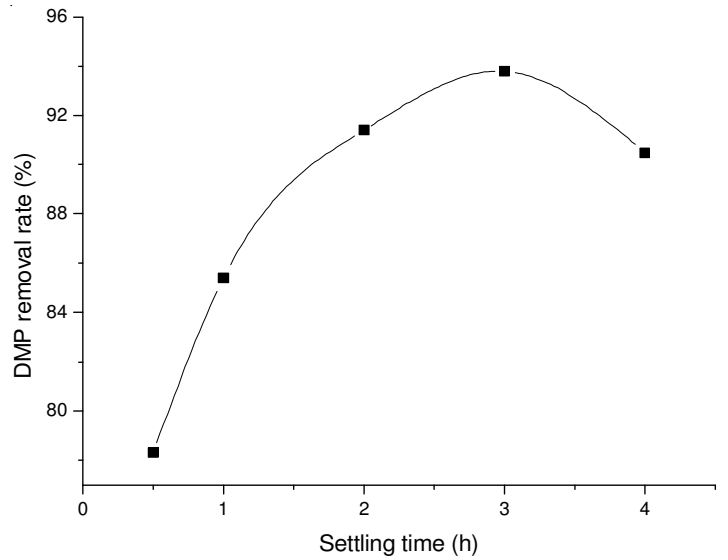

Fig. 5. Effect of settling on removal of DMP

Effect of molecular weight on removal rate of DMP: The effect of the molecular weight on the removal efficiency of DMP was investigated with the molecular weight from 0.57 $\times 10^{7}-1.28 \times 10^{7}$ and not adjust the $\mathrm{pH}$ value. Fig. 6 showed that the removal efficiency of DMP increased gradually with molecular weight increased. The result indicated that the flocculant molecular weight was higher, the molecular of the flocculant would have the longer molecular chain and more positive charge, leading to the more favorable abilities of absorption/bridging and charge neutralization. Therefore, the adopted optimum molecular weight for DMP removal is $1.28 \times 10^{7}$.

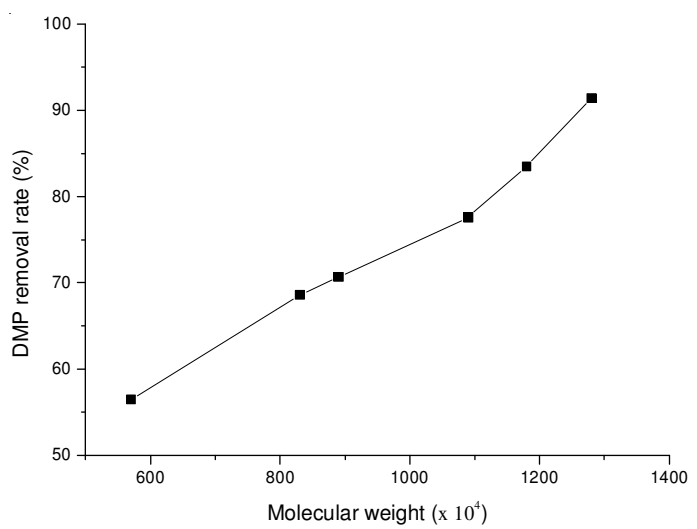

Fig. 6. Effect of molecular weight on removal of DMP

Analysis of variance (ANOVA): Table-2 showed the results of orthogonal table L9 $\left(3^{3}\right)$. Statistical testing of parameters was performed using Fisher's statistical test for ANOVA and results are presented in Table-3. Table- 3 shows the ANOVA results performed on the $\mathrm{L} 9\left(3^{3}\right)$ matrix. The dosage, settling time and initial $\mathrm{pH}$ were found to be insignificant at $95 \%$ confidence level by the F-test as shown in Table-3. In addition, the percentage contribution (PC \%) of each factor to the DMP 


\begin{tabular}{|c|c|c|c|c|c|}
\hline \multicolumn{6}{|c|}{$\begin{array}{c}\text { TABLE-2 } \\
\text { FIXED-BED SYSTEM WITH ORTHOGONAL TEST }\end{array}$} \\
\hline No. & A & $\mathrm{B}$ & $\mathrm{C}$ & Dummy factor & Removal efficiency of DMP (\%) \\
\hline 1 & 1 & 1 & 1 & 1 & 77.6 \\
\hline 2 & 1 & 2 & 2 & 2 & 85.4 \\
\hline 3 & 1 & 3 & 3 & 3 & 82.4 \\
\hline 4 & 2 & 1 & 2 & 3 & 86.8 \\
\hline 5 & 2 & 2 & 3 & 1 & 90.2 \\
\hline 6 & 2 & 3 & 1 & 2 & 85.2 \\
\hline 7 & 3 & 1 & 3 & 2 & 79.8 \\
\hline 8 & 3 & 2 & 1 & 3 & 88.0 \\
\hline 9 & 3 & 3 & 2 & 1 & 86.8 \\
\hline $\mathrm{k}_{1}$ & 81.800 & 81.400 & 83.600 & 83.767 & $\mathrm{y}=\frac{1}{9} \mathrm{~T}-84.32 ; \quad \mathrm{T}=758.9$ \\
\hline $\mathrm{k}_{2}$ & 87.400 & 87.867 & 85.233 & 83.467 & \\
\hline $\mathrm{k}_{3}$ & 83.767 & 83.700 & 84.133 & 85.733 & \\
\hline $\mathrm{R}$ & 5.600 & 6.467 & 1.633 & 2.266 & \\
\hline $\mathrm{Si}$ & 48.429 & 64.469 & 4.162 & 126.16 & \\
\hline Optimization & & & & & \\
\hline
\end{tabular}

TABLE-3

ORTHOGONAL ANALYSIS TABLE

\begin{tabular}{cccccc}
\hline Factor & Sum of squares (SS) & Degree of freedom & F value & F critical value $(\mathrm{P}<0.05)$ & Percentage contribution $(\%)$ \\
\hline A & 48.429 & 2 & 1.536 & 4.46 & 41.38 \\
B & 64.469 & 2 & 2.044 & 4.46 & 55.06 \\
C & 4.162 & 2 & 0.132 & 4.46 & 3.56 \\
Error & 126.16 & 8 & - & - & - \\
\hline
\end{tabular}

removal efficiency was investigated to dosage being $41.38 \%$, initial $\mathrm{pH}$ being $55.06 \%$ and settling time being $3.56 \%$. Furthermore, the result indicated little cross effects among three factors. Therefore, ANOVA results and percentage contribution indicated initial $\mathrm{pH}$ is the most significant influence on the flocculation process.

Orthogonal optimization analysis: In order to confirm the optimum conditions from the single factor experiment, the orthogonal array was used to determine optimum removal conditions. Table- 2 also showed the optimal combination in orthogonal experimental design was $\mathrm{A}_{2} \mathrm{~B}_{2} \mathrm{C}_{2}$, but the single factor experiment was not involved. So adding $\mathrm{A}_{2} \mathrm{~B}_{2} \mathrm{C}_{2}$ validation tests, the result showed DMP removal rate is $94.6 \%$. The result is higher than the single factor and orthogonal experiment. Therefore, it is indicated the optimum removal conditions from the orthogonal array are dosage at $9 \mathrm{mg} \mathrm{L}^{-1}$, initial $\mathrm{pH}$ value at 6 and settling time at $3 \mathrm{~h}$.

\section{Conclusion}

In this study, $\mathrm{P}(\mathrm{AM}-\mathrm{DAC}-\mathrm{DMC})$ was characterized with ${ }^{1} \mathrm{H}$ NMR and SEM analysis and the removal efficiency of DMP in water by flocculation was investigated. ${ }^{1} \mathrm{H}$ NMR spectrum indicated obtain a novel P(AM-DAC-DMC) flocculant. SEM images provided the apparent morphology of the flocculants, which suggested P(AM-DAC-DAC) had a good adsorptionbridging ability in removal of DMP from water. Absorptionbridging, charge neutralization and enmeshment have been very important role of removal of DMP in water. The optimization results showed optimum conditions for dosage, initial $\mathrm{pH}$ and settling time were $9 \mathrm{mg} \mathrm{L}^{-1}, 6$ and $3 \mathrm{~h}$, respectively. In addition, the removal rate of DMP reached a maximum value being $94.6 \%$.

\section{ACKNOWLEDGEMENTS}

The authors are grateful for the financial support provided by the National Natural Science Foundation of China (Project No.NSFC, 21177164; 51078366) and Research Foundation for Advanced Talents, Chongqing City Management College (Project No. 2013 kyqd09).

\section{REFERENCES}

1. T.F. de Oliveira, O. Chedeville, H. Fauduet and B. Cagnon, Desalination, 276, 359 (2011).

2. Y. Lu, F. Tang, Y. Wang, J.H. Zhao, X. Zeng, Q.F. Luo and L. Wang, J. Hazard. Mater., 168, 938 (2009).

3. W.E. Gledhill, R.G. Kaley, W.J. Adams, O. Hicks, P.R. Michael, V.W. Saeger and G.A. LeBlanc, Environ. Sci. Technol., 14, 301 (1980).

4. H.L. Zheng, P. Zhang, X.L. Deng, G.C. Zhu, C. Yang, W. Fan, C.J. Zhu and Z. Yang, CIESC J., 63, 279 (2012).

5. S. Na, Y.G. Ahn, M. Cui and J. Khim, J. Environ. Manage., 101, 104 (2012).

6. B. Osman, E.T. Özer, E. Demirbel, S. Güçer and N. Besirli, Sep. Purif. Technol., 109, 40 (2013).

7. C.E. Mackintosh, J. Maldonado, J. Hongwu, N. Hoover, A. Chong, M.G. Ikonomou and F.A. Gobas, Environ. Sci. Technol., 38, 2011 (2004).

8. Y. Zeng and J. Park, Colloid Surf. A, 334, 147 (2009).

9. L. Keith and W. Telliard, Environ. Sci. Technol., 13, 416 (1979)

10. M.M. Abdel daiem, J. Rivera-Utrilla, R. Ocampo-Pérez, J.D. MéndezDíaz and M. Sánchez-Polo, J. Environ. Manage., 109, 164 (2012).

11. P. Zhang and B.Z. Ren, Asian J. Chem., 25, 3966 (2013).

12. H.L. Zheng, Z.Z. Jiang, J.R. Zhu, M.Z. Tan, L. Feng, L.W. Liu and W. Chen, Desalin. Water Treat., 51, 5674 (2013).

13. G.C. Zhu, H.L. Zheng, Z. Zhang, T. Tshukudu, P. Zhang and X.Y. Xiang, Chem. Eng. J., 178, 50 (2011).

14. H.L. Zheng, J.R. Zhu, Z.Z. Jiang, F.Y. Ji, M.Z. Tan, Y.J. Sun, S.X. Miao and X.K. Zheng, Adv. Mater. Res., 414, 172 (2011).

15. J.R. Zhu, H.L. Zheng, Z.Z. Jiang, Z. Zhang, L.W. Liu, Y.J. Sun and T. Tshukudu, Desalin. Water Treat., 51, 2791 (2013).

16. J.R. Zhu, H.L. Zheng, Z. Zhang, Z.Z. Jiang, Q.Q. Guang, T.M. Zhou, D. $\mathrm{Li}$ and W. Chen, CIESC J., 63, 4019 (2012).

17. Y. Liu, C. Lin and Y. Wu, J. Hazard. Mater., 146, 255 (2007). 